\title{
KINEMATICS OF DISK STARS IN THE SOLAR NEIGHBOURHOOD
}

\author{
A.E. GÓMEZ ${ }^{1}$, S. GRENIER ${ }^{1}$, S. UDRY ${ }^{2}$, M. HAYWOOD ${ }^{1}$ \\ AND

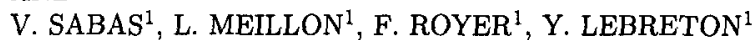 \\ 1 Observatoire de Paris, D.A.S.G.A.L., CNRS URA 335, 92195 Meudon Cedex, France \\ 2 Observatoire de Genève, 1290, Sauverny, Switzerland
}

Using Hipparcos parallaxes and proper motions together with radial velocity data and individual ages estimated from isochones, the velocity ellipsoid has been determined as a function of age. On the basis of the available kinematic data two different samples were considered: a first one (7789 stars) for which only tangential velocities were calculated and a second one containing 3104 stars with available $\mathrm{U}, \mathrm{V}$ and $\mathrm{W}$ velocity components and total velocities $\leq 65 \mathrm{~km} . \mathrm{s}^{-1}$.

The main conclusions are: -Mixing is not complete at about 0.8-1 Gyr. -The shape of the velocity ellipsoid changes with time getting rounder from $\sigma_{U} / \sigma_{V} / \sigma_{W}=1 / 0.63 / 0.42 \pm 0.04$ at about $1 \mathrm{Gyr}$ to $1 / 0.7 / 0.62 \pm 0.04$ at $4-5$ Gyr. -The age-velocity-dispersion relation (from the sample with kinematical selection) rises to a maximum, thereafter remaining roughly constant; there is no dynamically significant evolution of the disk after about 4-5 Gyr. -Among the stars with solar metallicities and $\log$ (age) $>9.8$ two groups are identified: one has typical thin disk characteristics, the other is older than $10 \mathrm{Gyr}$ and lags the LSR at about $40 \mathrm{~km} \cdot \mathrm{s}^{-1}$. -The variation of the tangential velocity with age (without selection on the tangential velocity) shows a discontinuity at about 10 Gyr, which may be attributed to stars typically of the thick disk populations for ages $>10$ Gyr.

\section{ON THE CHARACTERISTICS OF THE VELOCITY FIELD OF YOUNG STARS IN THE SOLAR NEIGHBOURHOOD}

\author{
J. TORRA ${ }^{1}$, A.E. GÓMEZ ${ }^{2}$, F. FIGUERAS ${ }^{1}$, F. COMERÓN ${ }^{3}$, S. GRENIER $^{2}$ \\ AND

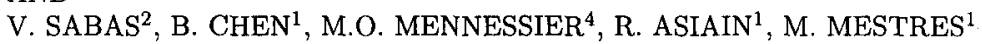 \\ 1 Universitat de Barcelona, Diagonal, 647, 08028 Barcelona, Spain \\ 2 Observatoire de Meudon, DASGAL/URA CNRS 335, 92195 Meudon Cedex, France \\ ${ }^{3}$ European Southern Observatory, Garching bei Munchen, Germany \\ ${ }^{4}$ Universite de Montpellier II, Pl. E. Bataillon, 34095 Montpellier Cedex 5, France
}

The analysis of stellar kinematics of the local system of young stars from Hipparcos data offers an excellent opportunity to revise models accounting for the origin of the observed deviations from circular motions, and to give new hints on the history of star formation and the dynamical processes involved in the evolution of our Galaxy. We have complemented the astrometric Hipparcos data with a careful compilation of available radial velocities and Strömgren photometry, information which enables us to obtain reliable space velocities and individual ages for a large number of stars. The velocity field has been studied by means of the classical first order approach, interpreting the trends observed in the Oort's contants in terms of the local expansion of the Gould's Belt (stars with ages less than 30-50 Myr, at heliocentric distances $\rho<400-600 \mathrm{pc}$ ). The fundamental geometric parameters of such structure have been determined. Several statistical methods have been applied to identify moving groups in the 4-dimensional space of (U,V,W,age). Our results definitively confirm their existence with mean velocity dispersions of the order of $5-7 \mathrm{~km} / \mathrm{s}$. The obtained results will be published in Astronomy \& Astrophysics. 